PROCEEDINGS OF THE

AMERICAN MATHEMATICAL SOCIETY

Volume 132, Number 3, Pages 919-927

S 0002-9939(03)07173-9

Article electronically published on September 12, 2003

\title{
CRITICAL EXPONENTS OF DISCRETE GROUPS AND $L^{2}-$ SPECTRUM
}

\author{
ENRICO LEUZINGER
}

(Communicated by Rebecca A. Herb)

\begin{abstract}
Let $G$ be a noncompact semisimple Lie group and $\Gamma$ an arbitrary discrete, torsion-free subgroup of $G$. Let $\lambda_{0}(M)$ be the bottom of the spectrum of the Laplace-Beltrami operator on the locally symmetric space $M=\Gamma \backslash X$, and let $\delta(\Gamma)$ be the exponent of growth of $\Gamma$. If $G$ has rank 1, then these quantities are related by a well-known formula due to Elstrodt, Patterson, Sullivan and Corlette. In this note we generalize that relation to the higher rank case by estimating $\lambda_{0}(M)$ from above and below by quadratic polynomials in $\delta(\Gamma)$. As an application we prove a rigiditiy property of lattices.
\end{abstract}

\section{INTRODUCTION}

In the study of geometric aspects of discrete subgroups of semisimple Lie groups the main interest has been in lattices (a lattice is a discrete subgroup $\Gamma$ of a Lie group $G$ such that the coset space $\Gamma \backslash G$ has finite Haar measure). Indeed, lattices enjoy several remarkable properties. For instance, in higher rank Lie groups, lattices are strongly rigid in the sense of G. D. Mostow, super-rigid in the sense of G. A. Margulis and, in particular, arithmetic (see [15, [17] and 25]). On the other hand, there are various interesting classes of discrete subgroups of semisimple Lie groups that are not lattices. First of all there is the elaborated theory of Kleinian groups (i.e., discrete subgroups of $P S L(2, \mathbb{C})$ ). These groups are closely related to the geometry and topology of three-manifolds and often admit interesting deformations (see e.g. 24]). In the higher rank case there are, for instance, the generalized Schottky groups constructed by Y. Benoist, the groups considered by V. G. Kac and E. B. Vinberg and those studied by G. A. Margulis and G. A. Soifer (see [3], [12] and [16] respectively).

In contrast to the lattice case and the case of Kleinian groups, one knows only very little about discrete subgroups of infinite covolume in higher rank Lie groups. We mention the work of Y. Guivarc'h and J.-P. Conze on limit sets and that of P. Albuquerque and J.-F. Quint on Patterson-Sullivan measures (see e.g. 9], 6] and [1], [19, [20]).

In the following, $G$ denotes a connected, semisimple Lie group without compact factors and with trivial center. Let $\Gamma$ be an arbitrary discrete, torsion-free subgroup

Received by the editors November 9, 2002.

2000 Mathematics Subject Classification. Primary 22E40, 53C20, 53C35.

Key words and phrases. Discrete subgroups of semisimple Lie groups, critical exponent, $L^{2}$ spectrum, locally symmetric spaces. 
of $G$. For a maximal compact subgroup $K$ of $G, X=G / K$ is a Riemannian symmetric space of noncompact type on which $G$ and $\Gamma$ act by isometries. Since $\Gamma$ is torsion-free, the quotient manifold $M=\Gamma \backslash X$ is a locally symmetric space.

In this note we study two numerical quantities which measure the size of such groups and manifolds. The first one, the critical exponent (or exponent of growth) of a discrete group $\Gamma \subset G$, is defined as

$$
\delta(\Gamma):=\limsup _{R \rightarrow \infty} \frac{\log n_{R}}{R} \geq 0
$$

where $n_{R}$ is the number of points in some orbit $\Gamma \cdot x \subset X$ which are contained in a ball of radius $R$ about $x$ (using the triangle inequality it is easy to see that $\delta(\Gamma)$ does not depend on the chosen point $x \in X)$. The critical exponent of $\Gamma$ thus measures the exponential growth rate of $\Gamma$-orbits in $X$. This rate is bounded from above by the volume growth rate of $X$ (also called volume entropy). The latter is equal to $2\|\rho\|$, where $2 \rho$ is the sum of positive restricted roots counted with multiplicity (see Section 2). Examples of subgroups $\Gamma$ with minimal critical exponent, $\delta(\Gamma)=0$, are, for instance, discrete abelian subgroups generated by semisimple elements of $G$. Lattices in $G$ have maximal critical exponent $\delta(\Gamma)=2\|\rho\|$ (see Section 2 where further examples are discussed).

The second quantity that we are going to consider is $\lambda_{0}(M)$, the bottom of the spectrum of the Laplace-Beltrami operator on the locally symmetric space $M=$ $\Gamma \backslash X$.

If the (real) rank of the semisimple group $G$ is 1 , then the above two quantities are known to be equivalent. More precisely, the following assertion holds:

Theorem (Elstrodt-Patterson-Sullivan-Corlette). If $\operatorname{rank}_{\mathbb{R}} G=1$, then

$$
\begin{aligned}
& \lambda_{0}(M)=\|\rho\|^{2} \quad \text { if } 0 \leq \delta(\Gamma) \leq\|\rho\| \text { and } \\
& \lambda_{0}(M)=\delta(\Gamma)(2\|\rho\|-\delta(\Gamma)) \text { if }\|\rho\| \leq \delta(\Gamma) \leq 2\|\rho\| .
\end{aligned}
$$

For Fuchsian groups these equations have been proved by J. Elstrodt [8] and S. Patterson [18, for discrete groups of isometries in higher-dimensional hyperbolic spaces the result is due to D. Sullivan [23, and for discrete subgroups of semisimple Lie groups of rank one to K. Corlette [7].

The goal of this note is to generalize the previous theorem to the higher rank case by estimating $\lambda_{0}(M)$ from above and below by quadratic polynomials in $\delta(\Gamma)$. Our main result (compare Theorem 1) is the following.

Theorem A. Let $\Gamma$ be a torsion-free, discrete subgroup of a semisimple Lie group $G$ without compact factors and with trivial center. Then the following estimates hold:

$$
\begin{aligned}
& \text { if } \delta(\Gamma) \in\left[0, \rho_{m}\right], \text { then } \lambda_{0}(M)=\|\rho\|^{2} ; \\
& \text { if } \delta(\Gamma) \in\left[\rho_{m},\|\rho\|\right] \text {, then }\|\rho\|^{2}-\left(\delta(\Gamma)-\rho_{m}\right)^{2} \leq \lambda_{0}(M) \leq\|\rho\|^{2} \text { and } \\
& \text { if } \delta(\Gamma) \in[\|\rho\|, 2\|\rho\|] \text {, then } \\
& \quad \max \left\{0 ;\|\rho\|^{2}-\left(\delta(\Gamma)-\rho_{m}\right)^{2}\right\} \leq \lambda_{0}(M) \leq\|\rho\|^{2}-(\delta(\Gamma)-\|\rho\|)^{2} \text {. }
\end{aligned}
$$

As an application we obtain the following characterization of lattices among all discrete subgroups of a given semisimple group $G$ that has Kazhdan's property (T) (see Theorem 2 for a more precise statement). 
Theorem B. Let $\Gamma$ be a torsion-free, discrete subgroup of a semisimple Lie group $G$ without compact factors and with trivial center. If $G$ has Kazhdan's property $(T)$, then $\Gamma$ is a lattice iff $\delta(\Gamma)=2\|\rho\|$.

Notation. We write $f \approx g$ for two real-valued functions $f$ and $g$ if there is a constant $C \geq 1$ such that $\frac{1}{C} f(x) \leq g(x) \leq C f(x)$ for all $x$.

\section{CRitical EXPonents}

2.1. Definitions. Let $d$ denote the distance function associated to a $G$-invariant Riemannian metric on $X=G / K$. The Poincaré series of a discrete subgroup $\Gamma$ of $G$ is defined as

$$
P_{s}(x, y):=\sum_{\gamma \in \Gamma} e^{-s d(x, \gamma y)}, \quad x, y \in X, s \in \mathbb{R} .
$$

It is then well known that, for any $x, y \in X, P_{s}(x, y)$ converges for $s>\delta(\Gamma)$ and diverges for $s<\delta(\Gamma)$ (see e.g. 22]).

Let $\mathfrak{g}$ be the Lie algebra of the semisimple group $G$, let $\mathfrak{g}=\mathfrak{k} \oplus \mathfrak{p}$ be a Cartan decomposition, and let $\mathfrak{a}$ be a maximal abelian subalgebra in $\mathfrak{p}$. Let further $\Sigma^{+}$ denote the set of positive restricted roots of the pair $(\mathfrak{g}, \mathfrak{a})$ with respect to some Weyl chamber $\mathfrak{a}^{+}$(see e.g. [10], Chapter VI, for definitions). We also denote by $m_{\alpha}=\operatorname{dim} \mathfrak{g}_{\alpha}$ the multiplicity of $\alpha \in \Sigma^{+}$and set $\rho:=\frac{1}{2} \sum_{\alpha \in \Sigma^{+}} m_{\alpha} \alpha \in \mathfrak{a}^{*}$. Since $\mathfrak{g}$ is semisimple the Killing form $\langle$,$\rangle is nondegenerate and defines an isomorphism$ $\mathfrak{a}^{*} \simeq \mathfrak{a} ; \alpha \mapsto \vec{\alpha}$ by $\langle\vec{\alpha}, H\rangle:=\alpha(H)$ for all $H \in \mathfrak{a}$; finally, we set $\langle\alpha, \beta\rangle:=\langle\vec{\alpha}, \vec{\beta}\rangle$.

2.2. The upper bound for critical exponents. The volume of a ball of radius $R$ about a point $x$ in a symmetric space $X$ of noncompact type and rank $r$ is given by

$$
\operatorname{vol} B_{R}(x) \approx R^{\frac{r-1}{2}} e^{2\|\rho\| R}
$$

see, e.g., 11] for a proof. Thus

$$
\limsup _{R \rightarrow \infty} \frac{1}{R} \log \operatorname{vol} B_{R}(x)=2\|\rho\| \text {. }
$$

For any discrete subgroup $\Gamma$ we have $n_{R}=\left|B_{R}(x) \cap \Gamma \cdot y\right| \leq$ const. vol $B_{R}(x)$. We thus conclude from the definition and the above approximation that $\delta(\Gamma) \leq 2\|\rho\|$.

\subsection{Some examples.}

2.3.1. Abelian groups of hyperbolic isometries. Let $G=K A N$ be an Iwasawa decomposition of the semisimple Lie group $G$. Pick elements $a_{1}, \ldots, a_{m}$ of $A$ with $m \leq r=\operatorname{rank}(X)$ such that $\log a_{1}, \ldots, \log a_{m} \in \mathfrak{a}$ are linearly independent. If $\Gamma$ is the discrete subgroup of $G$ generated by $a_{1}, \ldots, a_{m}$, then $\delta(\Gamma)=0$. To see this note that the orbit of $x \in X=G / K$ under $A$ is a totally geodesic flat submanifold. Thus the growth of $\Gamma \subset A$ in $X$ is the same as that in the flat $A x$, i.e., Euclidean: $n_{R} \approx R^{m}$. Hence for these groups, $\delta(\Gamma)$ attains the minimal possible value. 
2.3.2. Discrete groups containing unipotent elements. If $\Gamma \subset G=S L(n, \mathbb{R})$ contains unipotent elements, then $\delta(\Gamma)>c(G)>0$ where $c(G)$ is some universal constant.

Proof. Let $e \neq u \in \Gamma$ be unipotent and let $\Gamma^{*}:=\langle u\rangle \subset \Gamma$ be the infinite cyclic group generated by $u$. Since it is clear from that definition that $\delta\left(\Gamma^{*}\right) \leq \delta(\Gamma)$, it suffices to prove the assertion for $\Gamma^{*}$. For $G=S L(n, \mathbb{R})$ and $X=S L(n, \mathbb{R}) / S O(n)$ we have the well-known facts

$$
d(g x, x) \approx d_{G}(g, e) \approx \log (1+\|g-e\|) \text { and }\left\|u^{m}\right\| \approx|m|
$$

see, e.g., 14. We thus get

$$
\sum_{m=-\infty}^{\infty} e^{-s d\left(u^{m} x, x\right)} \approx \sum_{k=0}^{\infty} 2 e^{-s a \log (1+b k)}=\sum_{k=0}^{\infty} 2(1+b k)^{-a s}
$$

for some positive constants $a$ and $b$. Hence $0<\frac{1}{a}=\delta\left(\Gamma^{*}\right) \leq \delta(\Gamma)$.

2.3.3. Generalized Schottky groups. These groups have been constructed by Y. Benoist in 3]. As an abstract group a generalized Schottky group $\Gamma_{g}$ is isomorphic to a free group with $g$ generators (we assume in the following that the set $S$ of generators is symmetric, i.e., $s \in S$ implies $s^{-1} \in S$ and thus $|S|=2 g$ ). Let $\Gamma_{g}$ be endowed with the word metric $d_{\Gamma}$, which is defined as follows: $d_{\Gamma}(e, \gamma)=l$ if $\gamma$ can be written as a minimal word of the form $\gamma=s_{1} \cdots s_{l}$ with $s_{i} \in S$.

We choose a base point $x_{0}$ in $X$, and we set $c(g):=\max _{s \in S} d_{X}\left(x_{0}, s x_{0}\right)$. For $R \in \mathbb{R}^{+}$, denote by $n_{R}^{\Gamma}$ (resp. $n_{R}^{X}$ ) the number of $\gamma \in \Gamma_{g}$ with $d_{\Gamma}(e, \gamma) \leq R$ (resp. $d_{X}\left(x_{0}, \gamma x_{0}\right) \leq R$ ). Then we have, for $\gamma \in \Gamma_{g}$ and $k \in \mathbb{N}$, firstly that $n_{k}^{\Gamma}=2 g(2 g-1)^{k-1} ;$ and secondly that

$$
\begin{aligned}
d_{X}\left(x_{0}, \gamma x_{0}\right)= & d_{X}\left(x_{0}, s_{1} \cdots s_{l} x_{0}\right) \leq d_{X}\left(x_{0}, s_{1} x_{0}\right)+d_{X}\left(s_{1} x_{0}, s_{1} \cdots s_{l} x_{0}\right) \\
& =d_{X}\left(x_{0}, s_{1} x_{0}\right)+d_{X}\left(x_{0}, s_{2} \cdots s_{l} x_{0}\right) \leq c(g)+d_{X}\left(x_{0}, s_{2} \cdots s_{l} x_{0}\right) .
\end{aligned}
$$

Hence by induction $d_{X}\left(x_{0}, \gamma x_{0}\right) \leq c(g) l=c(g) d_{\Gamma}(e, \gamma)$ and therefore

$$
n_{k}^{\Gamma}=2 g(2 g-1)^{k-1} \leq n_{k c(g)}^{X} .
$$

Eventually we thus get the following lower bound for the critical exponent of a generalized Schottky group:

$$
\delta\left(\Gamma_{g}\right)=\limsup _{R \rightarrow \infty} \frac{\log n_{R}^{X}}{R} \geq \frac{1}{c(g)} \log (2 g-1)>0 .
$$

2.3.4. Zariski-dense discrete subgroups. A discrete Zariski-dense subgroup $\Gamma$ of a noncompact semisimple Lie group satisfies $0<\delta(\Gamma)$ (see, e.g., 1], Proposition 4.7).

2.3.5. Lattices. If $\Gamma$ is a lattice in $G$, then $\delta(\Gamma)=2\|\rho\|$. This is proved in [1], Thm. 7.4. For lattices the critical exponent thus attains the maximal possible value (compare 2.2). 


\section{The BotTom of The $L^{2}$-SPECTRUM}

3.1. Definition. We define the Laplace-Beltrami operator (see [5] and 21]) first on $C^{\infty}$-functions with compact support on the locally symmetric space $M=\Gamma \backslash X$ by $\Delta_{M}:=-\operatorname{div}$ grad. This operator has a unique selfadjoint extension to $L^{2}(M)$, which we also denote by $\Delta_{M}$. Let $\operatorname{Spec} \Delta_{M}$ denote the $L^{2}$-spectrum of $\Delta_{M}$ and define the bottom of the spectrum $\lambda_{0}(M):=\inf \operatorname{Spec} \Delta_{M} \geq 0$. Notice that the closed set $\operatorname{Spec} \Delta_{M}$ is contained in the interval $\left[\lambda_{0}(M), \infty\right)$.

3.2. A characterization of $\lambda_{0}(M)$. The following proposition has been proved by K. Corlette (see [7], Section 4).

Proposition 1. (Corlette) (a) The Green function (or resolvent kernel) $g^{\lambda}$ for the resolvent $\left(\Delta_{M}-\lambda I\right)^{-1}$ can be written in the form

$$
g^{\lambda}(\check{x}, \check{y})=\sum_{\gamma \in \Gamma} G^{\lambda}(x, \gamma y), \quad \check{x}, \check{y} \in M=\Gamma \backslash X
$$

where $G^{\lambda}$ is the Green function for $\left(\Delta_{X}-\lambda I\right)^{-1}$ on $X=G / K$ and $x$ (resp. $y$ ) in $X$ is a lift of $\check{x}$ (resp. $\check{y}$ ).

(b) The bottom $\lambda_{0}(M)$ of the $L^{2}$-spectrum of $M$ is the supremum of all $\lambda \in$ $\left[0,\|\rho\|^{2}\right]$ for which the right-hand side of $(*)$ converges for all $x \neq y$.

In order to state the next proposition, we need some additional notation. Let $G=K \overline{A^{+}} K$ be a Cartan decomposition, and let $x_{0}$ be the base point of the globally symmetric space $X$ corresponding to the maximal compact subgroup $K$ of $G$. Any element $g \in G$ can be written as $g=k \exp H k^{\prime}$ where $H \in \overline{\mathfrak{a}^{+}}$is uniquely determined by $g$ (see [10], Ch. IX.1).

For any $x, y$ in the homogeneous space $X=G / K$, there are $g, h \in G$ such that $x=g x_{0}$ and $y=h x_{0}$. For $\gamma \in \Gamma \subset G$ we then have $d(x, \gamma y)=d\left(x_{0}, g^{-1} \gamma h x_{0}\right)$ and $g^{-1} \gamma h=k \exp H k^{\prime}$. The isometries $g$ and $h$ are not unique; if we also have $x=\bar{g} x_{0}$ and $y=\bar{h} x_{0}$, then $\bar{g}=g k_{1}, \bar{h}=h k_{2}$ for $k_{1}, k_{2} \in K$. But since $\bar{g}^{-1} \gamma \bar{h}=$ $k_{1}^{-1} k \exp H k^{\prime} k_{2}$ the element $H$ is well defined and (for $x$ and $y$ fixed) will be denoted by $H(\gamma)$ in what follows. In conclusion we thus have

$$
d(x, \gamma y)=d\left(x_{0}, k \exp H(\gamma) k^{\prime} x_{0}\right)=d\left(x_{0}, \exp H(\gamma) x_{0}\right)=\|H(\gamma)\| .
$$

Notice that the last equality holds since $A x_{0}$ is totally geodesic in $X$.

Let $\Sigma^{++} \subset \Sigma^{+}$be the set of indivisible positive roots (i.e., those positive roots $\alpha$ for which $\frac{1}{2} \alpha$ is not a root). The next proposition has been proved by J.-P. Anker and L. Ji (see [2], 4.2.).

Proposition 2. (Anker-Ji) For any $\lambda<\|\rho\|^{2}$ and any $H \in \overline{\mathfrak{a}^{+}}$with $\|H\|$ sufficiently large, the Green function $G^{\lambda}$ on the symmetric space $X$ of rank $r$ satisfies

$$
G^{\lambda}\left(\exp H x_{0}, x_{0}\right) \approx \prod_{\alpha \in \Sigma^{++}}\{1+\alpha(H)\}\|H\|^{\frac{1-r}{2}-\left|\Sigma^{++}\right|} e^{-\rho(H)-\|H\| \sqrt{\|\rho\|^{2}-\lambda}} .
$$

The following proposition characterizes the bottom of the $L^{2}$-spectrum.

Proposition 3. Let $\Gamma$ be a torsion-free, discrete subgroup of a noncompact, semisimple Lie group $G$. Then the bottom $\lambda_{0}(M)$ of the $L^{2}$-spectrum of the locally symmetric space $M=\Gamma \backslash G / K$ is the supremum of all $\lambda \in\left[0,\|\rho\|^{2}\right]$ such that the 
series

$$
s^{\lambda}(\check{x}, \check{y}):=\sum_{\gamma \in \Gamma} \prod_{\alpha \in \Sigma^{++}}\{1+\alpha(H(\gamma))\}\|H(\gamma)\|^{\frac{1-\gamma}{2}-\left|\Sigma^{++}\right|} e^{-\rho(H(\gamma))-\|H(\gamma)\| \sqrt{\|\rho\|^{2}-\lambda}}
$$

converges for some lifts $x$ (resp. $y$ ) to $X$ of $\check{x}$ (resp. $\check{y}$ ) in $M$ and where $H(\gamma)$ is the unique element in $\overline{\mathfrak{a}^{+}}$such that $d\left(x_{0}, \exp H(\gamma) x_{0}\right)=d(x, \gamma y)$.

Proof. Since $\Delta_{X}$ commutes with isometries, one has for all $x, y \in X$ that $G^{\lambda}(g x, g y)$ $=G^{\lambda}(x, y)$ for all $g \in G$. In particular, we have (using the same notation as above)

$$
\begin{aligned}
G^{\lambda}(x, \gamma y)=G^{\lambda}\left(g x_{0}, \gamma h x_{0}\right)= & G^{\lambda}\left(x_{0}, g^{-1} \gamma h x_{0}\right) \\
& =G^{\lambda}\left(x_{0}, k \exp H(\gamma) k^{\prime} x_{0}\right)=G^{\lambda}\left(x_{0}, \exp H(\gamma) x_{0}\right) .
\end{aligned}
$$

Since the convergence of the series $(*)$ in Proposition 1(a) depends only on the asymptotic behaviour of $G^{\lambda}$ for $x$ and $y$ far away from one another, the claim follows from Proposition 2.

3.3. Relation to the critical exponent. Using the definitions in 2.1 we denote by $\overline{\mathfrak{a}^{+}}(1)$ the set of all unit vectors in the closed Weyl chamber $\overline{\mathfrak{a}^{+}}$. We define $\rho_{m}:=\min _{H \in \overline{\mathfrak{a}^{+}(1)}} \rho(H)$. Since $\vec{\rho} \in \mathfrak{a}^{+}$(see [4], Ch. VI, 1.10, Prop. 29) we have $\max _{H \in \overline{\mathfrak{a}^{+}}(1)} \rho(H)=\|\rho\|$. We then get $\rho_{m}\|H(\gamma)\| \leq \rho(H(\gamma)) \leq\|\rho\|\|H(\gamma)\|$ for all $\gamma \in \Gamma$. We can now state our main result which estimates $\lambda_{0}(M)$ in terms of $\delta(\Gamma)$.

Theorem 1. Let $\Gamma$ be a torsion-free, discrete subgroup of a semisimple Lie group $G$ without compact factors and with trivial center. Then the following estimates hold:

$$
\begin{aligned}
& \text { if } \delta(\Gamma) \in\left[0, \rho_{m}\right] \text {, then } \lambda_{0}(M)=\|\rho\|^{2} ; \\
& \text { if } \delta(\Gamma) \in\left[\rho_{m},\|\rho\|\right] \text {, then }\|\rho\|^{2}-\left(\delta(\Gamma)-\rho_{m}\right)^{2} \leq \lambda_{0}(M) \leq\|\rho\|^{2} \text { and } \\
& \text { if } \delta(\Gamma) \in[\|\rho\|, 2\|\rho\|] \text {, then } \\
& \qquad \max \left\{0 ;\|\rho\|^{2}-\left(\delta(\Gamma)-\rho_{m}\right)^{2}\right\} \leq \lambda_{0}(M) \leq\|\rho\|^{2}-(\delta(\Gamma)-\|\rho\|)^{2} \text {. }
\end{aligned}
$$

Proof. Since $\alpha(H(\gamma)) \geq 0$ for all $\alpha \in \Sigma^{++}$and all $H(\gamma)$, we have

$$
1 \leq \prod_{\alpha \in \Sigma^{++}}\{1+\alpha(H(\gamma))\}
$$

Moreover, if we fix some (large) radius $R_{0}$, we have for $\|H(\gamma)\| \geq R_{0}$ also the estimate

$$
\prod_{\alpha \in \Sigma^{++}}\left\{\frac{1+\alpha(H(\gamma))}{\|H(\gamma)\|}\right\} \leq \prod_{\alpha \in \Sigma^{++}}\left\{\frac{1}{R_{0}}+c_{\alpha}\right\}=: C
$$

where $c_{\alpha}:=\max _{H \in \overline{\mathfrak{a}^{+}}(1)} \alpha(H)>0$.

We next set $s_{\min }(\lambda):=\rho_{m}+\sqrt{\|\rho\|^{2}-\lambda}$ and $s_{\max }(\lambda):=\|\rho\|+\sqrt{\|\rho\|^{2}-\lambda}$. The series in Proposition 3 and the above two estimates then yield the inequalities

$$
\sum_{\gamma \in \Gamma}\|H(\gamma)\|^{a} e^{-s_{\max }(\lambda)\|H(\gamma)\|} \leq s^{\lambda}(\check{x}, \check{y}) \leq C \sum_{\gamma \in \Gamma}\|H(\gamma)\|^{b} e^{-s_{\min }(\lambda)\|H(\gamma)\|}
$$

with $a=\frac{1-r}{2}-\left|\Sigma^{++}\right|$and $b=\frac{1-r}{2}$. For $k \in \mathbb{N}$, let $r_{k}$ denote the number of $\Gamma$-orbit points of $x_{0}$ lying in a half-open shell of radii in $\left(k-\frac{1}{2}, k+\frac{1}{2}\right]$ centered at $x_{0}$. Using 
the notation from Section 2 and observing that $\|H(\gamma)\|=d(x, \gamma y)$, we can write the above inequalities also in the following form:

$$
\sum_{k=0}^{\infty} r_{k} k^{a} e^{-s_{\max }(\lambda) k} \leq s^{\lambda}(\check{x}, \check{y}) \leq C \sum_{k=0}^{\infty} r_{k} k^{b} e^{-s_{\min }(\lambda) k}
$$

with $\lambda \leq\|\rho\|^{2}$. For the following note that the series $\sum_{k=0}^{\infty} r_{k} k^{\beta} e^{-s k}, \quad \beta \in \mathbb{R}$ converges for $s>\delta(\Gamma)$ and diverges for $s<\delta(\Gamma)$.

Step $(\mathrm{a}): \delta(\Gamma) \leq \rho_{m}$. We have $s_{\min }(\lambda)>\rho_{m} \geq \delta(\Gamma)$ for all $\lambda \in\left(-\infty,\|\rho\|^{2}\right)$. Since the Poincaré series $P_{s}(x, y)$ converges for any $s>\delta(\Gamma)$, the right-hand side of $(* *)$ and hence $s^{\lambda}(\check{x}, \check{y})$ converges for all $\lambda<\|\rho\|^{2}$ and therefore, by Proposition $3, \lambda_{0}(M)=\|\rho\|^{2}$.

Step (b): $\delta(\Gamma) \geq \rho_{m}$. Since the series $s^{\lambda}(\check{x}, \check{y})$ in Proposition 3 diverges for any $\lambda>\lambda_{0}(M)$, the right-hand side of $(* *)$ diverges for any $\lambda>\lambda_{0}(M)$. From the definition of the critical exponent we then get

$$
\begin{aligned}
\delta(\Gamma) \geq & s_{\min }\left(\lambda_{0}(M)\right) \Longleftrightarrow 0 \leq \delta(\Gamma)-\rho_{m} \geq \sqrt{\|\rho\|^{2}-\lambda_{0}(M)} \\
& \Longleftrightarrow\left(\delta(\Gamma)-\rho_{m}\right)^{2} \geq\|\rho\|^{2}-\lambda_{0}(M) \Longleftrightarrow \lambda_{0}(M) \geq\|\rho\|^{2}-\left(\delta(\Gamma)-\rho_{m}\right)^{2} .
\end{aligned}
$$

Step $(\mathrm{c}): \delta(\Gamma) \geq\|\rho\|$. Since the series $s^{\lambda}(\check{x}, \check{y})$ converges for any $\lambda<\lambda_{0}(M)$, the left-hand side of $(* *)$ converges for any $\lambda<\lambda_{0}(M)$. The definition of the critical exponent then yields that

$$
\begin{aligned}
\delta(\Gamma) \leq s_{\max }\left(\lambda_{0}(M)\right) \Longleftrightarrow(\delta(\Gamma)-\|\rho\|)^{2} \leq & \|\rho\|^{2}-\lambda_{0}(M) \\
& \Longleftrightarrow \lambda_{0}(M) \leq\|\rho\|^{2}-(\delta(\Gamma)-\|\rho\|)^{2} .
\end{aligned}
$$

This completes the proof of Theorem 1 .

In the special case where $\operatorname{rank}_{\mathbb{R}} G=1(=\operatorname{rank} X)$ we have $\rho_{m}=\|\rho\|$ and Theorem 1 thus reduces to the following.

Corollary 1. (Elstrodt-Patterson-Sullivan-Corlette) If $\operatorname{rank}_{\mathbb{R}} G=1$ and $\Gamma$ and $M$ are as in Theorem 2, then

$$
\begin{aligned}
& \lambda_{0}(M)=\|\rho\|^{2} \quad \text { if } 0 \leq \delta(\Gamma) \leq\|\rho\| \quad \text { and } \\
& \lambda_{0}(M)=\delta(\Gamma)(2\|\rho\|-\delta(\Gamma)) \quad \text { if }\|\rho\| \leq \delta(\Gamma) \leq 2\|\rho\| .
\end{aligned}
$$

\section{A RIGIDITy PHENOMENON FOR LATTICES}

In the rank one case, K. Corlette proved the following remarkable gap or rigidity theorem (see [7], Thm. 4.4):

Theorem (Corlette). Let $\Gamma$ be a discrete subgroup of the isometry group of a quaternionic hyperbolic space (resp. the Cayley hyperbolic plane) that is not a lattice. Then the critical exponent of $\Gamma$ is $\leq 4 n$ (resp. $\leq 16)$.

Notice that for the above hyperbolic spaces $2\|\rho\|$ (which by 2.3 .5 is equal to the critical exponent of lattices) equals $4 n+2$ (resp. 22). That result of Corlette is extended to the higher rank case by the following theorem, which characterizes lattices among discrete subgroups for all those symmetric spaces whose isometry groups have Kazhdan's property (T). It is well known that a connected, semisimple Lie group has property $(\mathrm{T})$ if and only if it has no simple factors locally isomorphic to the rank one groups $S O(n, 1)$ or $S U(n, 1)$ (see [15], [25]). 
Theorem 2. Let $G$ be a semisimple Lie group $G$ without compact factors and with trivial center. Let $\Gamma$ be a discrete, torsion-free subgroup of $G$ that is not a lattice. If $G$ has Kazhdan's property $(T)$, then there is a constant $c^{*}(G)>0$ depending on $G$ but not on $\Gamma$ such that $\delta(\Gamma) \leq 2\|\rho\|-c^{*}(G)$.

Proof. The assumption that $G$ has property (T) implies that there is a constant $c(G)>0$ depending on $G$ but not on $\Gamma$ such that $0<c(G) \leq \lambda_{0}(\Gamma \backslash X)$ (see [13, Thm. 2). We may assume that $\delta(\Gamma) \geq\|\rho\|$. Then by Theorem 1 we have $0<$ $c(G) \leq\|\rho\|^{2}-(\delta(\Gamma)-\|\rho\|)^{2}$ and thus $\delta(\Gamma) \leq \sqrt{\|\rho\|^{2}-c(G)}+\|\rho\|=: 2\|\rho\|-c^{*}(G)$ with $c^{*}(G)>0$.

\section{REFERENCES}

[1] P. Albuquerque, Patterson-Sullivan theory in higher rank symmetric spaces, Geom. Funct. Anal. 9 (1999), 1-28. MR 2000d:37021

[2] J.-P. Anker AND L. Ji, Heat kernel and Green function estimates on noncompact symmetric spaces, Geom. Funct. Anal. 9 (1999), 1035-1091. MR 2001b:58038

[3] Y. Benoist, Propriétés asymptotiques des groupes linéaires, Geom. Funct. Anal. 7 (1997), 1-47. MR 98b:22010

[4] N. Bourbaki, Éléments de mathématique, Groupes et Algèbres de Lie, Chapitres IV, V, VI, Paris, 1981. MR 83g:17001

[5] I. Chavel, Eigenvalues in Riemannian Geometry, Pure and Applied Mathematics, Vol. 115, Academic Press, Orlando, FL, 1984. MR 86g:58140

[6] J.-P. Conze and Y. Guivarc'H, Densité d'orbites d'actions de groupes linéaires, in: Rigidity in Dynamics and Geometry, M. Burger and A. Iozzi (eds.), Springer-Verlag, Berlin, 2002. MR 2003b:00029

[7] K. Corlette, Hausdorff dimensions of limit sets. I, Invent. Math. 102 (1990), 521-541. MR 91k:58067

[8] J. Elstrodt, Die Resolvente zum Eigenwertproblem der automorphen Formen in der hyperbolischen Ebene. Teil I. Math. Ann. 203 (1973), 295-330, Teil II. Math. Z. 132 (1973), 99-134, Teil III. Math. Ann. 208 (1974), 99-132. MR 50:12922a MR 50:12922b, MR 50:12922c

[9] Y. Guivarc'H, Produits de matrices aléatoires et applications aux propriétés géométriques des sous-groupes du groupe linéaire, Ergodic Theory Dynam. Systems 10 (1990), 483-512. MR 92c:60011

[10] S. Helgason, Differential Geometry, Lie Groups, and Symmetric Spaces, Pure and Applied Mathematics, Vol. 80, Academic Press, New York, 1978. MR 80k:53081

[11] G. KNIEPER, On the asymptotic geometry of nonpositively curved manifolds, Geom. Funct. Anal. 7 (1997) 755-782. MR 98h:53055

[12] V. G. Kac And E. B. Vinberg, Quasi-homogeneous cones, Math. Notes 1 (1967), 231-235; translated from Mat. Zametki 1 (1967), 347-354. MR 34:8280

[13] E. LEuZinger, Kazhdan's property (T), $L^{2}$-spectrum and isoperimetric inequalities for locally symmetric spaces, Comment. Math. Helv. 78 (2003), 116-133.

[14] A. Lubotzky, S. Mozes, and M. S Raghunathan, The word and Riemannian metrics on lattices of semisimple groups, Inst. Hautes Études Sci. Publ. Math. 91 (2000), 5-53. MR 2002e: 22011

[15] G. A. Margulis, Discrete Subgroups of Semisimple Lie Groups, Ergebnisse der Mathematik und ihrer Grenzgebiete, Vol. 17, Springer-Verlag, New York, 1991. MR 92h:22021

[16] G. A. Margulis And G. A. Soifer, Maximal subgroups of infinite index in finitely generated linear groups, J. of Algebra 69 (1981), 1-23. MR 83a:20056

[17] G. D. Mostow, Strong Rigidity of Locally Symmetric Spaces, Ann. Math. Stud. 78 (1978). MR 52:5874

[18] S. Patterson, The limit set of a Fuchsian group, Acta Math. 136 (1976), 241-273. MR 56:8841

[19] J.-F. Quint, Mesures de Patterson-Sullivan en rang supérieur, Geom. Funct. Anal. 12 (2002), $776-809$ 
[20] J.-F. Quint, Divergence exponentielle des sous-groupes discrets en rang supérieur, Comment. Math. Helv. 77 (2002), 563-608. MR 2003j:22015

[21] R. Strichartz, Analysis of the Laplacian on the complete Riemannian manifold, J. Funct. Anal. 52 (1983), 48-79. MR 84m:58138

[22] D. Sullivan, The density at infinity of a discrete group of hyperbolic motions, Inst. Hautes Études Sci. Publ. Math. 50 (1979), 171-202. MR 81b:58031

[23] D. Sullivan, Related aspects of positivity in Riemannian geometry, J. Differential Geom. 25 (1987), 327-351. MR 88d:58132

[24] W. P. Thurston, The Geometry and Topology of 3-Manifolds, Princeton Lecture Notes, 1978.

[25] R. Zimmer, Ergodic Theory and Semisimple Groups, Monographs in Mathematics, Vol. 81, Birkhäuser, Boston, 1984. MR 86j:22014

Math. Institut II, Universität Karlsruhe, D-76128 Karlsruhe, Germany

E-mail address: Enrico.Leuzinger@math.uni-karlsruhe.de 